\title{
How Much is too Much on Monitoring Tasks? Visual Scan Patterns of Single Air Traffic Controller Performing Multiple Remote Tower Operations
}

\author{
Wen-Chin $\mathrm{Li}^{1}$, Peter Kearney ${ }^{2}$, Graham Braithwaite ${ }^{1}$ and John J. H. Lin ${ }^{3}$ \\ ${ }^{1}$ Safety and Accident Investigation Centre, Cranfield University, Cranfield, \\ Bedfordshire, U.K. \\ ${ }^{2}$ ATM Operations and Strategy, Irish Aviation Authority, 11-12 D’olier Street, \\ Dublin, Ireland \\ ${ }^{3}$ Office of Institutional Research, National Central University, Taiwan.
}

\section{Corresponding author: Wen-Chin Li}

Postal Address: Safety and Accident Investigation Center, Cranfield University, Martell House, Cranfield, Bedfordshire, MK43 0TR, United Kingdom

Tel: +44 (1234) 758527

E-mail:wenchin.li@cranfield.ac.uk

\section{Content of Manuscript}

Running Head: Multiple Remote Towers Operated by Single ATCO

Manuscript Type: Research Article

Exact Word Count of Text: 6,423 words

Abstract: 207 words

Table: 1

Figures: 9

References: 58 


\title{
How Much is too Much on Monitoring Tasks? Visual Scan Patterns of Single Air Traffic Controller Performing Multiple Remote Tower Operations
}

\begin{abstract}
The innovative concept of multiple remote tower operation (MRTO) is where a single air traffic controller (ATCO) provides air traffic services to two or more different airports from a geographically separated virtual Tower. Effective visual scanning by the air traffic controller is the main safety concern for human-computer interaction, as the aim of MRTO is a single controller performing air traffic management tasks originally carried out by up to four ATCOs, comprehensively supported by innovative technology. Thirty-two scenarios were recorded and analyzed using an eye tracking device to investigate the above safety concern and the effectiveness of multiple remote tower operations. The results demonstrated that ATCOs' visual scan patterns showed significant task related variation while performing different tasks and interacting with various interfaces on the controller's working position (CWP). ATCOs were supported by new display systems equipped with pan tilt zoom (PTZ) cameras allowing enhanced visual checking of airport surfaces and aircraft positions. Therefore, one ATCO could monitor and provide services for two airports simultaneously. The factors influencing visual attention include how the information is presented, the complexity of that information, and the characteristics of the operating environment. ATCO's attention distribution among display systems is the key human-computer interaction issue in single ATCO performing multiple monitoring tasks.
\end{abstract}

Keywords: Air Traffic Management; Aviation Safety; Cost-Efficiency; Human-Computer Interactions; Multiple Remote Tower Operations; Situation Awareness 


\section{Introduction}

The initial concept of remote tower operation (RTO) was for air traffic services (ATS) to be delivered remotely without direct observation from a local tower (Kraiss \& Kuhlen, 1996). Based on the concept of remote tower operations, multiple remote tower operations (MRTO) offers further opportunity for cost efficiency of air traffic services for small and medium sized airports, especially if a single controller could provide air traffic services to two (or more airports) at the same time. Remote tower technology allows one air traffic controller (ATCO) to control one or more airports at the same time, a significant consideration of course are the appropriate traffic volumes for a single air traffic controller to manage (SESAR Joint Undertaking, 2013, 2015). ATCOs use Out the Window (OTW) visualization media supported by radar data processing (RDP), electronic flight strips (EFS) and a voice communications network (VCS) to provide air traffic services (Moehlenbrink \& Papenfuss, 2011). This Multiple Remote Towers research project was sponsored by the Single European Sky ATM Research Program (SESAR) and the ATM Operations Division of the Irish Aviation Authority. The Remote Tower Centre (RTC) was located at Dublin Air Traffic Services Unit, 150 miles away from both Shannon and Cork airports where the services were provided simultaneously. Cork airport handled approximately 50,242 movements in 2016 and Shannon airport handled approximately 25,059 movements in 2016 (Irish Aviation Authority, 2017).

The innovative concept of Multiple Remote Tower Operations (MRTO) is principally suitable for lower traffic density airports. The visual cues and objects which ATCOs routinely use for safe operations must be provided by the surveillance cameras; the data-communication links and the systems must support the provision of air traffic services at two (or more) different airfields simultaneously (Van Schaik, Roessingh, Lindqvist, \& Falt, 2016). Groundbreaking technology enables precise image-video resolution for signal detection and recognition. The crucial factor to assure aviation safety is the cooperative interaction between the human and the technical systems being used (Onken \& Walsdorf, 2001). ATCO's visual attention and situation awareness are the main safety concerns of human-computer interaction in MRTO, as the expectation of MRTO is for 'a single ATCO to perform the tasks originally designed to be executed by up to four ATCOs'. Therefore, the development of enhanced video resolution for remote air traffic services is not sufficient, it must integrate human-centered design in MRTO systems (Friedrich \& Mohlenbrink, 2013; Kearney, Li, Braithwaite, \& Greaves, 2017). 


\section{Objectives of Single European Sky ATM Operational Steps}

The EU Single European Sky initiative (SES) was introduced to restructure European airspace and propose innovative measures for air traffic management to achieve the objectives of enhanced cost-efficiency and improved airspace and airport capacity whilst simultaneously improving safety performance. The main driver of the implementation of the remote tower concept is cost-efficiency and the safety criteria to be applied should ensure that the level of safety after the introduction into service of the remote tower concept is at least not reduced compared to current conventional tower operations (European Aviation Safety Agency, 2015a). Many air navigation service providers (ANSPs) have developed automated systems using video-panorama cameras for synthetic outside views (Leitner \& Oehme, 2016). Research into remote tower operations increased over the last 20 years (European Aviation Safety Agency, 2014; SESAR Joint Undertaking, 2015). The emerging technology of RTO developed slowly during the initial stages but in recent times has taken a leap forward with virtual tower operations based on EUROCAE WG-100 standard "Remote and Virtual Towers" (EUROCAE, 2016). Research on multiple remote tower operations directly contributes to the objectives of the simultaneous provision of remote Air Traffic Services for multiple aerodromes. It is outlined in the Operational Improvement Step (OIS) SDM-0205 linked to SESAR Work Package (WP) 06.09.03 of the EU ATM Master Plan. This activity falls under SESAR Operational Step 3 for multiple remote tower operations (figure 1).

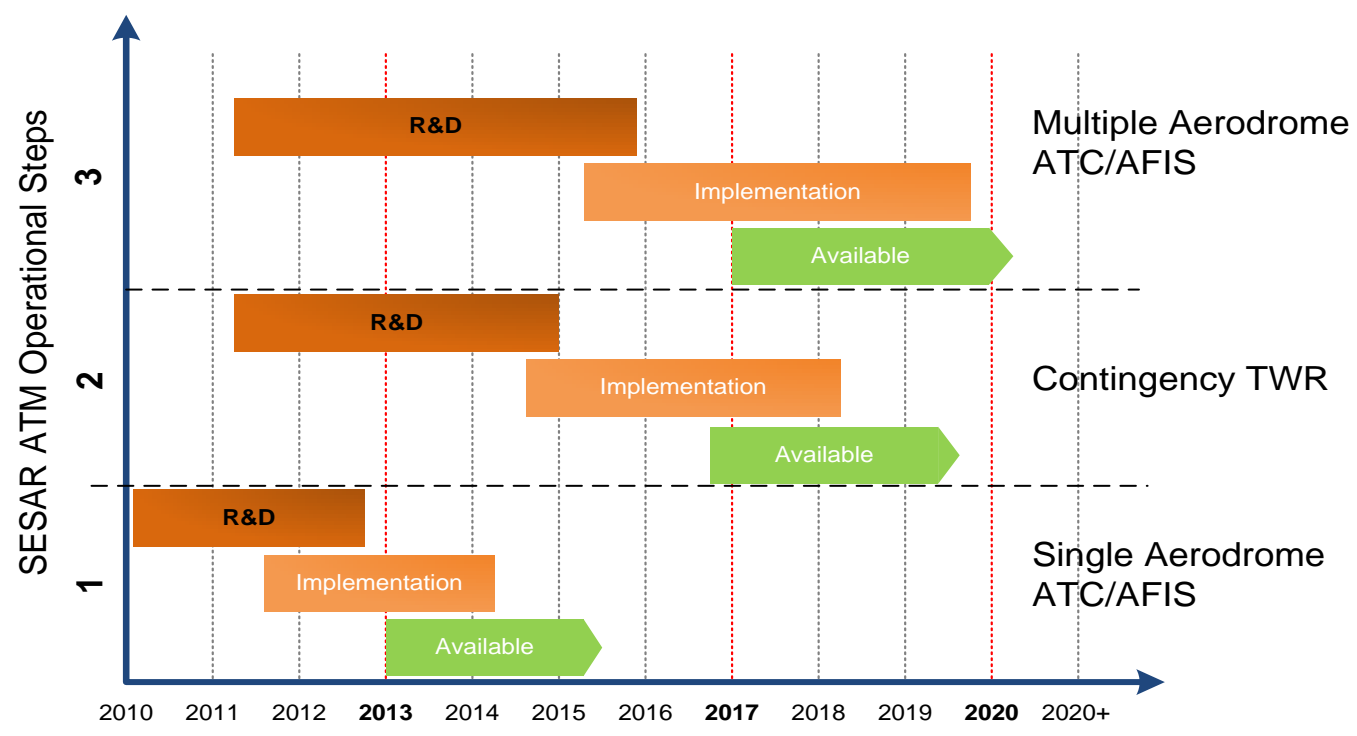

Figure 1. The Timelines of SESAR ATM Operational Steps for Single Aerodrome, Contingency Tower and Multiple Aerodrome ATC/AFIS 


\subsection{The Development of Multiple Remote Tower Operations}

Innovative systems development requires careful assessment of human information processing at the initial design stage to assure effective operators' situation awareness, safety and cost-efficiency (Chang, Yang, \& Hsiao, 2016; Honn, Satterfield, McCauley, Caldwell, \& Dongen, 2016). The cognitive match between an ATCO's information processing and external information presentation is a key requirement for effective monitoring performance in multiple remote tower operations. A well-designed interface should provide sufficient cues to rapidly direct the operator's visual scanning to desired objects with the least fixation duration. Therefore, highlighting the importance of the design of controller's working position (CWP) for presenting information via Out the Window visualization (OTW), Radar Data Processing (RDP), Electronic Flight Strips (EFS) and a Communications Network (VCS) to provide air traffic services, which is an emergent theme of human-computer interactions on MRTO (Hollan, Hutchins, \& Kirsh, 2000).

The US NextGen program (Federal Aviation Administration, 2012) also investigates the diverse aspects of tower control including human-computer interaction, situation awareness, cost of airport control tower, safety management and capacity variation. Similarly, NASA has examined remote tower operations for improving runway safety (Dorighi \& Rabin, 2002). Preliminary research found that RTO can provide substantial economic benefits compared with traditional operations of local physical air traffic control towers, as NextGen proposed an innovative concept to address airport capacity problems by introducing more integrated tower information, providing weather conditions and surveillance data as well as decision support tools to ATCOs (Nene, 2008). The results of human-in-the-loop experiments demonstrated that the concept of remote tower operations exhibited encouraging improvements in communications and departure rates with no differences in perceived workload, effort, safety and situation awareness (Nickelson, Jones, \& Zimmerman, 2011). Learnings from multiple remote tower operations are not only beneficial in understanding the performance of innovative systems but will also assist in how these advanced systems can impact on safety, capacity and cost-efficiency (Irish Aviation Authority, 2016; Van Lancker, Mondelaers, Wauters, \& Van Huylenbroeck, 2016).

\subsection{Interface Design Impact to ATCOs' Cognitive Processes}

Working with advanced automated systems, human operators not only have to monitor multiple displays with efficient distributed attention, but they must also intervene if automation fails by relocating their attention to the area requiring 
immediate attention (Carmen Bruder, 2014). The path of visual attention can reveal the cognitive process of human-computer interaction between operators and machines (Allsop \& Gray, 2014; Kearney, Li, \& Lin, 2016). Therefore, an operator's eye movements on the displays can reveal human information processes and how the interface design impacts operator's performance (Goldberg \& Kotval, 1999). For example, saccades (rapid movements between fixations) may reflect the operator's direction of an attention shift (Katoh, 1997; Kowler, 2011; Salvucci \& Goldberg, 2000), the distribution of one's fixations on an interesting area is related to attention allocation (Henderson, 2003), and can facilitate mechanisms to construct situation awareness (Johnson \& Proctor, 2004). In this way, an ATCO's eye movement parameters can be treated as a window into the cognitive system, allowing interface designers to capture ATCO's cognitive process (Henderson, 2003). Pupil dilation increases as a function of cognitive demand. ATCO's are constantly scanning the progress of aircraft in order to provide a safe separation and expeditious service. Observing ATCOs' eye movement patterns reveals that pupil dilation after alert activation is significantly bigger than before alert activation (Kearney, Li, \& Lin, 2016).

Visual attention is a precursor to initiating the cognitive process involved in attention distribution, situation awareness, and real-time decision-making (Lavine, Sibert, Gokturk, \& Dickens, 2002). Since the air traffic management system in Europe often operates to its limits, new operational concepts and technologies are constantly required to enhance capacity, safety and cost-efficiency. Future ATM systems must increase capacity and improve safety standards while at the same time deliver economic improvements (Muller, Giesa, \& Anders, 2001). The duration of human visual scanning is more related to processing complexity than to visual search efficiency (Robinski \& Stein, 2013), as much more time is spent in fixations than in saccades. A saccade amplitude is computed from the sum of the distances between consecutive fixations with the units of pixels or visual angle degree between each successive fixation (Goldberg \& Kotval, 1999). That means the more saccade amplitude deployed in visual scans on an specific display, the more attentions distributed to the instrument related to task performance (Katoh, 1997). On the other hand, saccade amplitude could be an index to observe if the interface design increases operator's cognitive process or not (Liversedge \& Findlay, 2000). In addition, effective saccades play an important role in scanning the elements, which could in turn be used to identify whether the elements being scanned are relevant or irrelevant based on the saccade velocity (McColemana \& Blair, 2013; Remington, Wu, \& Pashler, 2011). It appears that saccade velocity might be associated with how fast the operator's attention shifts and cognitive process (K. Rayner, 1998). 


\subsection{Visual Parameters Related to Human-Computer Interaction}

The path of fixations is associated with selective attention and accurate judgments for perceptual targets (Henderson, 2003). Saccadic eye movements are controlled by top-down visual processes, which are coordinated closely with perceptual attention (Zhao, Gersch, Schnitzer, Dosher, \& Kowler, 2012). This indicates that saccadic paths are intentional and meaningful, and are based on the requirements of the task and trajectory prediction to the near future (Kowler, 2011). However, Wickens et al. (2001) proposed that attention allocation is determined by the bottom-up capture of salient stimulus, inhibited by the effort required to move the focus of attention, and driven by the expectancy of seeing valuable stimulus in the traffic environment. To apply eye tracking technology in the context of monitoring tasks, it is necessary to understand the pattern of ATCO's monitoring is reflected by eye movements, such as how ATCOs guide their eye movements during monitoring phases and how eye scan patterns change during the monitoring process (Hasse \& Bruder, 2015). Most eye movements are in the form of saccades and fixations which are fast eye movements followed by a period of remaining relatively stationary in the same position. The features of slower saccade velocity over the relevant areas of interests (AOIs) could be associated closely with the knowledge-based visual scan process (Hoffman \& Subramaniam, 1995). AOIs were defined as ATCO's fixations gathered together closely on a specific display which suggests there is some information in the closeness of these fixations that attracts ATCO's attentions. Saccadic eye movements are proven as top-down visual processes relating to ATCO's perceptual attention (Zhao, Gersch, Schnitzer, Dosher, \& Kowler, 2012). This indicates that saccadic paths are intentional and meaningful, and are based on the requirements of the task in hand (Kowler, 2011).

ATCOs not only have to distribute their attention to detect potential conflicts among aircraft, both in the air and on the ground, but also have to monitor several radio frequencies to maintain situation awareness to prevent critical events. Condensed monitoring tasks and an augmented visual channel is foreseen as the most promising way to increase the capacity and safety of air traffic services (Beier \& Gemperlein, 2004). Visual parameters are related to different operational content ( $\mathrm{Yu}, \mathrm{Li}$, Wang, Braithwaite, \& Greaves, 2016), which could explore the interaction between human operator and the innovative technology of remote tower (Koenig \& Lachnit, 2011; Komogortsev \& Karpov, 2013). Monitoring performance is the most critical aspect related to safety in multiple remote tower operations. By applying eye tracking technology, ATCOs' eye movements and attention distributions can be investigated either bottom-up (stimulus-driven) or top-down (goal-driven) cognitive processes, the nature of the monitoring task will feed back to system design and ATCOs' training in 
the future. The eye tracking parameters are well suited for calculating the outcome of monitoring tasks (Hasse, Grasshoff \& Bruder, 2012). Based on literature reviews, there are four null hypotheses regarding ATCOs' visual parameters on performing MRTO which will be tested as follows,

1. $\mathrm{H}_{0}$ : ATCOs' fixation counts on the AOIs would have no significant interaction effect to perform MRTO tasks

2. $\mathrm{H}_{0}$ : ATCOs' fixation duration on the AOIs would have no significant interaction effect to perform MRTO tasks

3. $\mathrm{H}_{0}$ : ATCOs' saccade amplitude on the AOIs would have no significant interaction effect to perform MRTO tasks

4. $\mathrm{H}_{0}$ : ATCOs' pupil dilation on the AOIs would have no significant interaction effect to perform MRTO tasks

\section{Method}

\subsection{Scenarios}

The SESAR Safety Case for certification of multiple remote tower operations has distinct safety requirements for live trials. This approach outlines the activities of safety assessment to be conducted for the entire Multiple Remote Tower Systems including people, procedures, and equipment. Thirty-two scenarios were recorded using an eye tracking device to investigate human-computer interaction and use of the supporting camera systems of a single ATCO performing live exercises of multiple remote tower operations. The recordings consisted of tracking ATCOs' visual parameters across display systems while performing real and realistic multiple remote tower operations.

All scenarios contained three different air traffic control tasks: surface movement control (SMC), which is the air traffic control service provided to aircraft, vehicles and personnel on the maneuvering area of an aerodrome excluding the runway in use at both Shannon and Cork airports; air movement control (AMC) which is the air traffic control service provided to aircraft in the vicinity of an aerodrome and to aircraft, vehicles and personnel on the runway in use in at both Shannon and Cork airports; and SMC plus AMC involving both Shannon and Cork airport. The approval of the Cranfield University Research Ethic Committee was granted (CURES/1506/2016) in advance of the research taking place. All collected data were only available to the research team and stored in accordance to the United Kingdom Ethical Code and the Data Protection Act. 


\subsection{Apparatus}

Remote Tower Module (RTM): The RTM accommodates SMC and AMC working positions equipped with identical display systems including (1) the out of the window (OTW) visualization with fourteen active screens and one standby unit in the event of equipment failure. The displays match the Pan Tilt Zoom (PTZ) Camera resolution of 1920 x 1080 pixels with a refresh rate of $60 \mathrm{~Hz}$ in a 220 degrees configuration. These screens are sufficiently flexible to permit an ATCO to arrange the airports view to be split evenly between the two airports or if the operational situation requires, to have a larger view of a particular airport; (2) electronic flight strip (EFS) system which is divided into two parts; one for Shannon airport and one for Cork airport; (3) radar data processing (RDP) which can be used as a distance indicator to touch-down and is divided into two parts one for Shannon and one for Cork airport; (4) a voice communication system (VCS) which was equipped with a Schmid Communications Panel. It is used for both GND-AIR and GND-GND communications comprising all necessary frequencies and intercom direct dial buttons. These four interface displays on RTM are the areas of interest (AOIs) for human-computer interaction analysis in multiple remote tower operations. These four AOIs are the main sources of information related to ATCO's task performance. To increase ATCO's situation awareness, the borders of the display systems of OTW, RDP and EFS were distinguished by colors, Purple indicated Shannon airport, Green indicated Cork airport. The RTM is configured with the appropriate Shannon AMC/SMC and Cork AMC/SMC ATC VHF frequencies, the frequencies of Shannon on the top and the frequencies of Cork on the bottom. They are also colour coded to provide additional situation awareness to ATCOs (Purple for Shannon and Green for Cork, see figure 2).

Eye Tracking Device: A wearable and light-weight eye-tracking device "Pupil Pro" which consists of a headset including two cameras for eye movement data collection and analysis (Figure 2). The headset hosts two cameras, one facing the right eye of the participant (eye-camera) which has a resolution of 800 x 600 pixels and a frame rate of $60 \mathrm{~Hz}$, the other camera capturing the field of vision (world-camera) which has a resolution of $1920 \times 1080$ pixels with a frame rate of $60 \mathrm{~Hz}$. These two cameras can be synchronized after calibration. The 'world-camera' is mounted on the right top of the headset showing the orientation and view of the ATCO's view of the area of interests; the eye-camera is mounted offset right and low and is adjustable to suit different wearer's facial layout and track their pupil parameters accordingly (Kassner, Patera, \& Bulling, 2014). 


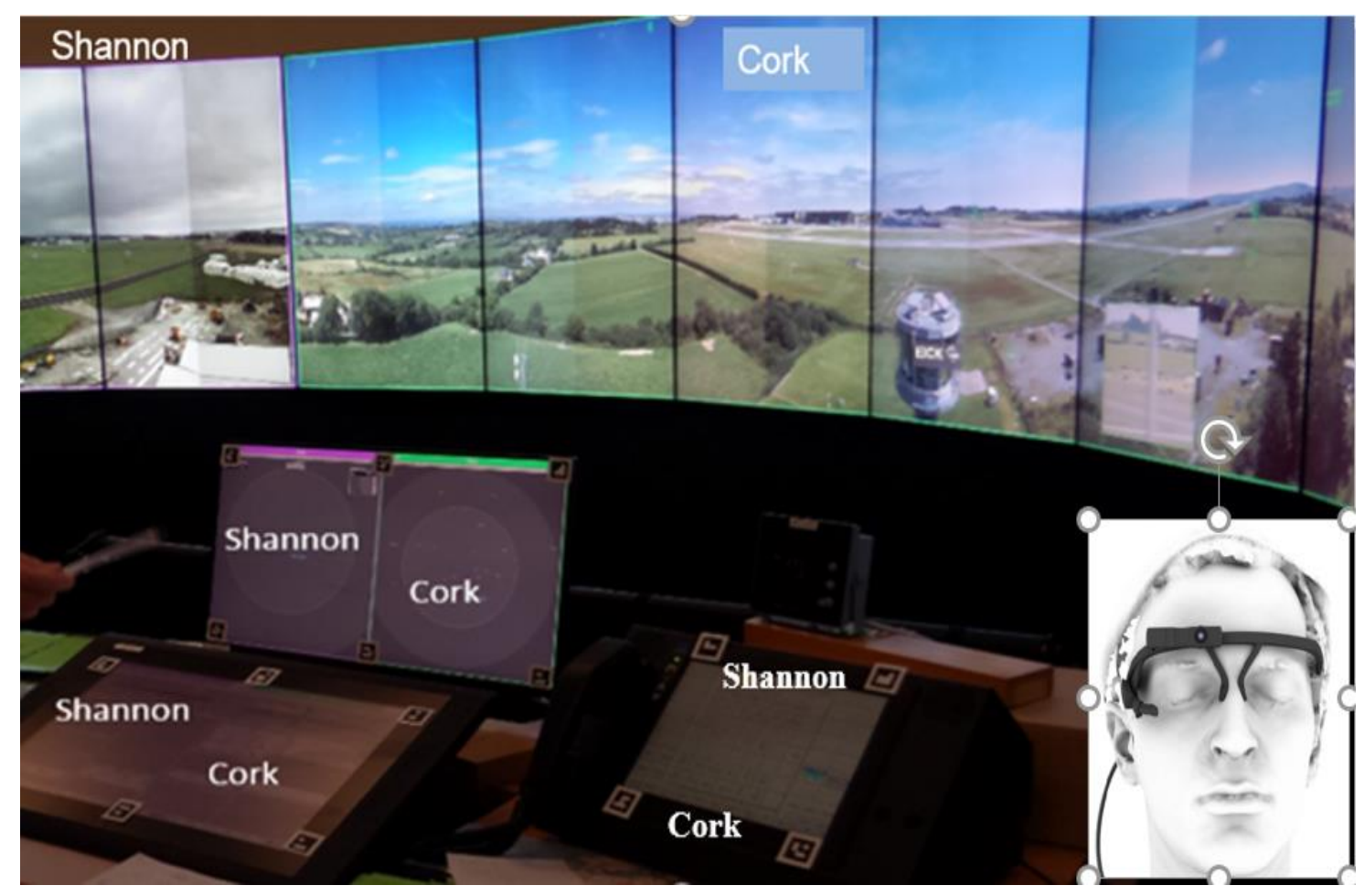

Figure 2. ATCO's using Pupil Pro Eye Tracker interacted with RTM comprised OTW, RDP, EFS and VCS (left-hand side are Shannon airport, right-hand side are Cork airport) for Multiple Remote Tower Operations.

\subsection{Research Design}

Thirty-two live exercises providing ATS for both Shannon and Cork airports from the remote tower control centre located at Dublin airport were conducted. The participants were all qualified ATCOs, holding operational licences for both Shannon and Cork airports. The assessment of human-computer interactions is based on ATCO's visual attention among AOIs while performing SMC and AMC tasks. Therefore, these 32 scenarios comprised three types of operation as between-subject variables, (1) SMC on both Shannon and Cork airports simultaneous; (2) AMC on both Shannon and Cork airports simultaneous; (3) AMC plus SMC on both Shannon and Cork airports simultaneous. The definition of 'simultaneous' is where the spacing between two aircraft arriving or departing at Shannon and Cork airports is less than that required if the two aircraft were landing or departing at the same airport. It means that the activities of AMC, SMC and AMC plus SMC on both Shannon airport and Cork airport are simultaneously being monitored and controlled by a single ATCO.

The eye tracking device collected and analysed ATCOs' visual parameters including fixation count, fixation duration, saccade amplitude and pupil dilation across the 
different interface displays (OTW, EFS, RDP \& VCS) on the RTM. The definition of fixation in this research is when the ATCO constantly maintains a gaze in a direction over 100 milliseconds. Due to different time frames for completing each scenario, all of 32 scenarios of eye tracker data are analysed for chunks of 60 seconds. The time frames of recorded eye movements contain the most critical visual parameters which reflect ATCO's cognitive processes and visual attention shifting among OTW, EFS, RDP and VCS. The research questions to be investigated by eye tracking data are:

- Will scanning be influenced in relation to task demands of MRTO?

- Will the ATCO's visual parameters vary while interacting with interfaces?

- Are the interfaces on CWP providing the necessary information to the ATCO for MRTO?

A period of 60 seconds for analysing ATCO's visual attention was supported by the consensus of experienced controllers. The live trial exercises related to remote control of over 500 live dynamic aircraft activities between Shannon and Cork airports. A project team was established to ensure that all aspects of relevant aviation activity were represented in the project. The project team consisted of a Project Manager, an ATM Specialist, a Human Factors Expert and two appropriately rated Controllers who were present for the live trials. To assure the safety of operations during the provision of service from the remote tower centre, both local towers were fully manned and operating in so-called shadow mode, capable of intervening in operations as required.

\section{Results}

The core concept of MRTO is to improve cost-efficiency and capacity of air traffic service, and maintain or improve the level of safety. The Air Navigation Service Provider and the Safety regulator's concerns on safety and human performance in respect of ATCO's attention distributions and operational performance had to be investigated before implementation. The complexity of MRTO involves organizing the traffic flow, providing information, and maintaining separation rules to both aircraft and ground vehicles between Shannon and Cork airports for SMC and AMC by interacting with OTW, EFS, RDP and VCS on monitoring tasks. The safety back-up of shadow operations from the physical towers at both Shannon and Cork airports never needed to intervene in the work of the single ATCO during the live demonstrations of multiple remote tower operations. When dealing with safety critical work contexts, the most suitable approach for cognitive processes assessment relies on unobtrusive techniques (Marchitto, Benedetto, Baccino \& Canas, 2016). This is the reason for 
applying eye tracking technology in this research. The results demonstrated that there are some substantial differences on ATCOs' visual parameters on different AOIs while performing AMS and SMC by remote tower module.

\subsection{Sample Characteristics}

Thirty-two scenarios of multiple remote tower operations included 11 SMC, 11 AMC and 10 SMC plus AMC were recorded by using an eye tracking device. ATCOs' eye movements across the displays on CWP including RDP, EFS, VCS and OTW were analyzed while performing SMC, AMC and SMC plus AMC at both Shannon and Cork airports. A series of mixed ANOVAs with AOIs (four levels: RDP, EFS, VCS, and OTW) as within-subject factor and operational tasks (three levels: SMC, AMC, and SMC+AMC) as between-subject factor were performed to assess single ATCO's eye movement patterns on human-computer interactions in multiple remote tower operations. The response variables are fixation count (FC), fixation duration (FD), saccade amplitude (SA), and pupil size (PS). The assumption of sphericity was verified by using Mauchly's test, and the Bonferroni was applied to perform pairwise comparisons after a significant overall test. Effect size of factors and interactions were quantified by partial eta square $\left(\eta_{p}^{2}\right)$. The descriptive statistics of sample characteristics were shown in table 1 .

Table 1. Descriptive statistics of mean and standard deviation of four eye movement parameters among three tasks and four AOIs

\begin{tabular}{|c|c|c|c|c|c|c|}
\hline Tasks & Visual & $\mathrm{N}$ & RDP & EFS & VCS & OTW \\
\hline & Parameters & & & & & \\
\hline \multirow{4}{*}{ SMC } & $\mathrm{FC}$ & 11 & $19.18(11.7)$ & $17.09(14.08)$ & $3.36(3.91)$ & $64.91(18.31)$ \\
\hline & FD & 11 & $.239(.079)$ & $.247(.105)$ & $.200(.202)$ & $.307(.084)$ \\
\hline & SA & 11 & $70.35(26.58)$ & $66.57(42.09)$ & $49.60(46.34)$ & $113.45(28.73)$ \\
\hline & PS & 7 & $76.22(3.41)$ & $69.81(9.12)$ & $81.01(6.04)$ & $75.99(3.74)$ \\
\hline \multirow{4}{*}{$\mathrm{AMC}$} & $\mathrm{FC}$ & 11 & $32.27(10.49)$ & $43.36(25.2)$ & $12.00(14.33)$ & $38.82(14.85)$ \\
\hline & FD & 11 & $.263(.041)$ & $.262(.060)$ & $.156(.137)$ & $.258(.038)$ \\
\hline & SA & 11 & 43.07(18.96) & $43.94(20.53)$ & $15.85(19.15)$ & $137.57(28.92)$ \\
\hline & PS & 7 & $84.91(4.65)$ & $83.70(10.41)$ & $94.64(22.09)$ & $85.86(10.83)$ \\
\hline \multirow{4}{*}{$\mathrm{SMC}+\mathrm{AMC}$} & $\mathrm{FC}$ & 10 & $23.50(16.64)$ & $56.80(39.28)$ & $2.20(3.82)$ & $26.40(19.95)$ \\
\hline & FD & 10 & $.339(.182)$ & $.238(.104)$ & $.154(.266)$ & $.303(.104)$ \\
\hline & SA & 10 & $52.60(25.17)$ & $36.00(34.01)$ & $20.81(41.54)$ & $141.00(38.43)$ \\
\hline & PS & 3 & $79.54(9.24)$ & $77.82(10.58)$ & $87.42(21.80)$ & $77.84(11.28)$ \\
\hline
\end{tabular}




\subsection{Fixation Counts (FC) among Interfaces on CWP for tasks performance}

There is a significant interaction between different interfaces (AOIs) and tasks, F $(3.20,46.40)=7.496, p<.001, \eta_{p}^{2}=.341$. A significant main effect of AOIs, $\mathrm{F}$ $(1.60,46.401)=23.205, p<.001, \eta_{p}^{2}=.445$ was found, but main effect of tasks is insignificant, $\mathrm{F}(2,29)=2.426, p=.106, \eta_{p}^{2}=.143$. Post-hoc comparison on AOI revealed that fixation counts on the communication system $\left(\mathrm{FC}_{\mathrm{VCS}}\right)$ are less significant than on the radar data $\left(\mathrm{FC}_{\mathrm{RDP}}\right)$, the strips $\left(\mathrm{FC}_{\mathrm{EFS}}\right)$, and the outside view (FC $<.001)$. Post-hoc comparison revealed $\mathrm{FC}_{\mathrm{SMC}}$ is smaller than $\mathrm{FC}_{\mathrm{AMC}}(p<.05)$ (figure 3). The results demonstrated that ATCOs exhibited the highest fixations numbers (64.9) at OTW and the lowest fixation numbers at VCS on SMC; however, EFS has the highest fixation numbers in both AMC (43.4) and AMC plus SMC (56.8). The SMC operation results in the highest useage of the outside camera view as indicated by the high fixations counts. The AMC operation scores high on flight strips and the outside camera (table $1 \&$ figure 3 ). Combining the two increases the useage of the strips to the highest levels, while the ATCO interaction with the camera system is decreased to the lowest levels. The controller is adapting task strategies to the situation and enhances their preparation by using flight strips data intensively while performing AMS plus SMC. Therefore, the first null hypothesis ' $\mathrm{H}_{0}$ : ATCOs' fixation counts on the AOIs would have no significant interaction effect to perform MRTO tasks' is rejected.

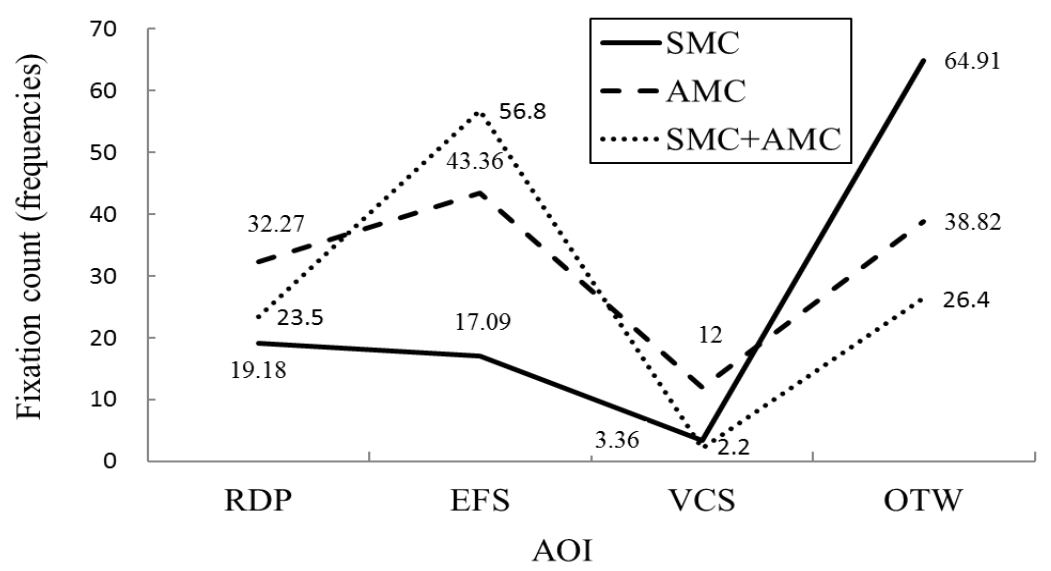

Figure 3. The differences of fixation count among AOIs and distinctive patterns of three tasks

\subsection{Fixation Duration (FD) among Interfaces on CWP for tasks performance}

There is no significant interaction between different interfaces (AOIs) and tasks, F 
$(3.702,53.680)=.705, \mathrm{p}>.05, \eta_{p}^{2}=.046$. A significant main effect of AOIs was found, $\mathrm{F}(1.851,53.680)=5.070, \mathrm{p}<.05, \eta_{p}^{2}=.149$, but no significant main effect on tasks, $\mathrm{F}(2,29)=.406, \mathrm{p}=.670, \eta_{p}^{2}=.027$. Post-hoc comparison on AOIs revealed FDVCS is smaller than FDRDP $(\mathrm{p}<.05)$ and FDOTW $(\mathrm{p}<.01)$ (figure 4). Fixation duration has a significant main effect on AOIs and revealed that ATCOs distributed the longest fixation duration on the RDP on both AMC (263 ms) and SMC plus AMC (339 ms). However, the longest fixation duration of SMC is on OTW (307 ms). Again, the shortest fixation duration is on VCS across three tasks (table $1 \&$ figure 4). The item of interest is held approximately stable on the retina during fixations with the majority between $154 \mathrm{~ms}$ and $339 \mathrm{~ms}$ depending on the complexity of information being processed and current cognitive load of ATCOs (table 1). Task and strategy related trends can be observed. Fixation duration is the lowest on the communication device. Combining SMC and AMC (high task load situation) results in longer fixation duration on radar data and similar levels for the using the cameras as during SMC. The effects are relatively small as fixations duration are bound by a minimum and in fact has no significant interaction between AOIs and tasks. Therefore, the second null hypothesis ' $\mathrm{H}_{0}$ : ATCOs' fixation duration on the AOIs would have no significant interaction effect to perform MRTO tasks' is accepted.

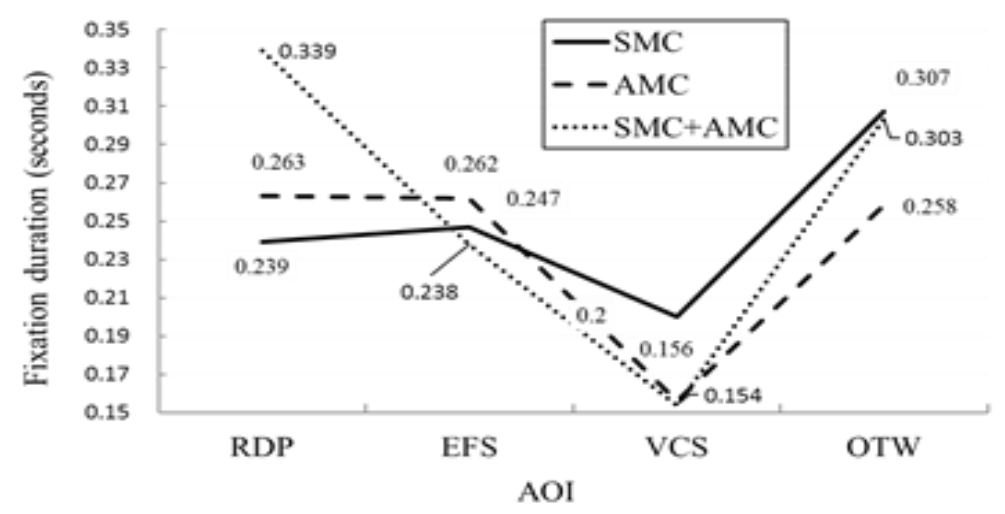

Figure 4. The differences of fixation duration among AOIs and distinctive patterns of three tasks

\subsection{Saccade Amplitude (SA) among Interfaces on CWP for tasks performance}

There is a significant interaction between different interfaces (AOIs) and tasks, $\mathrm{F}$ $(6,87)=2.437, \mathrm{p}<.05 \eta_{p}^{2}=.144$. A significant main effect of AOIs, F $(3,87)=$ $57.752, \mathrm{p}<.001, \eta_{p}^{2}=.666$ was found. A significant effect of tasks, $\mathrm{F}(2,29)=$ $3.578, \mathrm{p}<.05, \eta_{p}^{2}=.198$ was found as well. Post-hoc comparison on scenarios 
revealed participants' SASMC is higher than SAAMC $(\mathrm{p}<.05)$. Post-hoc comparison on AOIs revealed SAVCS is smaller than SARDP $(p<.01)$, SAEFS ( $p$ $<.05)$, and SAOTW $(\mathrm{p}<.001)$. Moreover, SARDP and SAEFS are smaller than SAOTW ( $<$.001) (figure 5). The VCS is the smallest saccade amplitude consistent with SMC (49.6 degree), AMC (15.85 degree) and SMC plus AMC (20.81 degree) (table 1) compared with EFS, OTW and RDP. The VCS is the smallest saccade amplitude consistent with SMC (49.6 degree), AMC (15.85 degree) and SMC plus AMC (20.81 degree) compared with EFS, OTW and RDP. However, the OTW is the highest saccade amplitude across SMC (113.45 degree), AMC (137.57 degree) and SMC plus AMC (141 degree) (table $1 \&$ figure 5). It revealed that OTW comprised of 14 visual reproduction display screens is a good human-centered design to facilitate ATCOs searching required information to perform MRTO. Therefore, the third null hypothesis is ' $\mathrm{H}_{0}$ : ATCOs' saccade amplitude on the AOIs would have no significant interaction effect to perform MRTO task' is rejected.

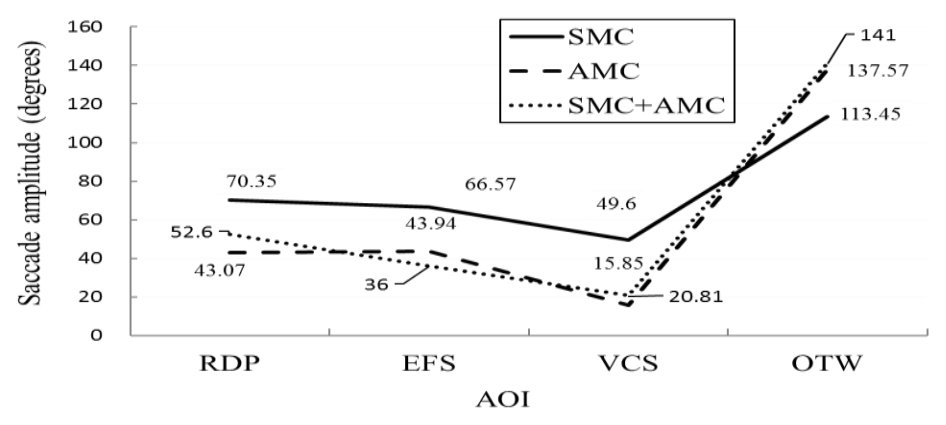

Figure 5. The differences of saccade amplitude among AOIs and distinctive patterns of three tasks

\subsection{Pupil Size (PS) among Interfaces on CWP for tasks performance}

Results indicated the interaction between different interfaces (AOIs) and tasks is not significant, $\mathrm{F}(3.05,21.35)=.307, \mathrm{p}>.05, \eta_{p}^{2}=.042$. A significant main effect of AOIs, F $(1.53,21.35)=5.790, \mathrm{p}<.001, \eta_{p}^{2}=.293$ was found, but no significant main effect on tasks, $\mathrm{F}(2,14)=2.765, \mathrm{p}=.097, \eta_{p}^{2}=.283$. Post-hoc comparison on AOIs revealed participants' PSVCS is higher than PSEFS $(p<.01)$ and PSOTW $(\mathrm{p}<.01)$. The results revealed that ATCO's pupil dilation has significant main effect on AOI (table $1 \&$ figure 6). The VCS is the highest pupil dilation consistent with AMC (94.64 pixels), SMC plus AMC (87.42 pixels) and 
SMC (81.01 pixels) compared with EFS, OTW and RDP. On the other hand, the EFS is the lowest pupil dilation across AMC (83.7 pixels), SMC plus AMC (77.82 pixels) and SMC (69.81 pixels). Therefore, the fourth null hypothesis is ' $\mathrm{H}_{0}$ : ATCOs' pupil dilation on the AOIs would have no significant interaction effect to perform MRTO tasks' is accepted.

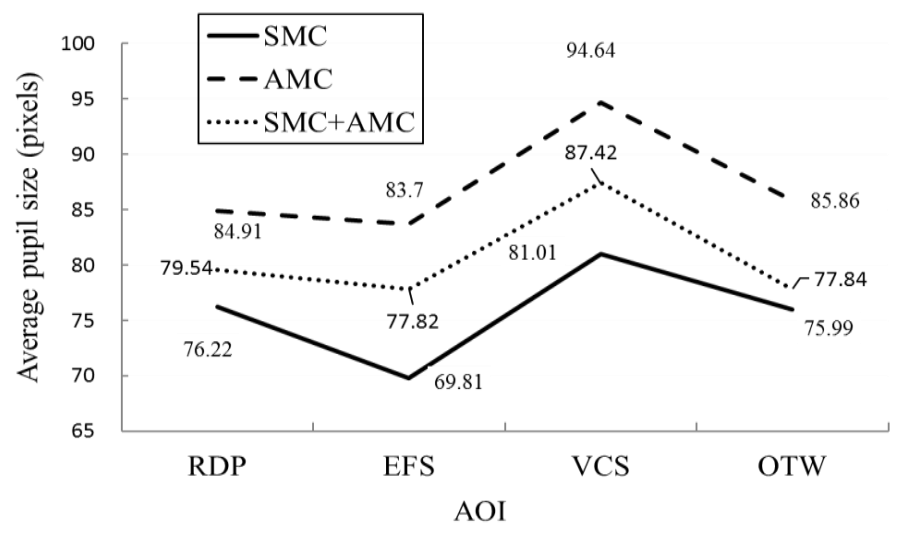

Figure 6. The differences of pupil size among AOIs and distinctive patterns of three tasks

\section{Discussion}

These live demonstrations of multiple remote tower operations represented all aspects of AMC and SMC including vehicle maneuvers and aircraft arriving and departing from Shannon and Cork airports. The result of this research has demonstrated that a single ATCO with the assistance of advanced technology is able to perform multiple remote tower tasks without compromising operational safety. Furthermore, ATCOs' eye movement parameters (fixation counts, fixation durations, saccade amplitude \& pupil dilation) can be measured in live operations and showed significant interactions effects between performing tasks (AMC, SMC or AMC plus SMC) and interfaces (EFS, OTW, RDP \& VCS) on CWP whilst conducting monitoring tasks for multiple remote tower operations. This research reflected that design of innovative air traffic management systems involving human-computer interactions requires an understanding of ATCO's cognitive processes and the operational deployment context in order that safety and capacity are not only maintained but enhanced (Langan-Fox, Canty \& Sankey, 2009). Previous visual science research found that increased challenge levels in tasks could influence operators' visual parameters such as increasing the frequency of long fixations (Van Orden, Limbert, Makeig, \& Jung, 
2001). The results of this research can provide a basis for future training and design for multiple remote tower operations.

\subsection{ATCO's Visual Scan Patterns related to Tasks Demanding}

The design of visual presentation on interface displays is the substantial factor to be considered from human-computer interactions and safety perspective for multiple remote tower operations. ATCOs tend to spend more time looking at interesting objects in the interface displays, as their fixations are drifting over the critical visual stimuli on the screens for tasks performance. The length of fixation duration can reflect difficulty in extracting information, and the number of fixations indicates the importance of the areas of interest (Kotval \& Goldberg, 1998). RTM provides detailed information which enables ATCOs to maintain continuous observation of all flight operations by using visual reproduction display screens (European Aviation Safety Agency, 2014, 2015b). It is an interesting finding and demonstrates that ATCOs distributed their fixations and shifted their attention in order to maintain situation awareness between two different airports based on the priority of the dynamic tasks (figure 7). Given that multiple remote tower is an innovative technology in the field of air traffic management, corresponding interface design should be evaluated so that ATCOs' workload could be minimized (Goldberg \& Kotval, 1999). The analysis of eye movement data found that ATCOs' scanning patterns were influenced by the performing tasks (SMC, AMC or SMC plus AMC). Multiple remote tower operations at two airports is achieved through the support of advanced technology, however increased visual monitoring tasks might induce perceived workload as a potential cost based on visual parameters. This is the reason both EFS and OTW have high percentages of fixation counts in these three different tasks, $79.2 \%$ at SMC, $65.3 \%$ at AMC and $77.4 \%$ at SMC plus AMC. SMC is focused on the ground movements of vehicles and aircraft, ATCOs relied heavily upon OTW by using PTZ to track the positions of aircraft and vehicles, therefore, the highest frequency of fixation (64.9) is on the SMC for both airports. VCS showed the lowest fixation counts across three tasks, as there is no ongoing operational requirement to switch the frequency of voice communication system once the appropriate frequencies are initially selected. 


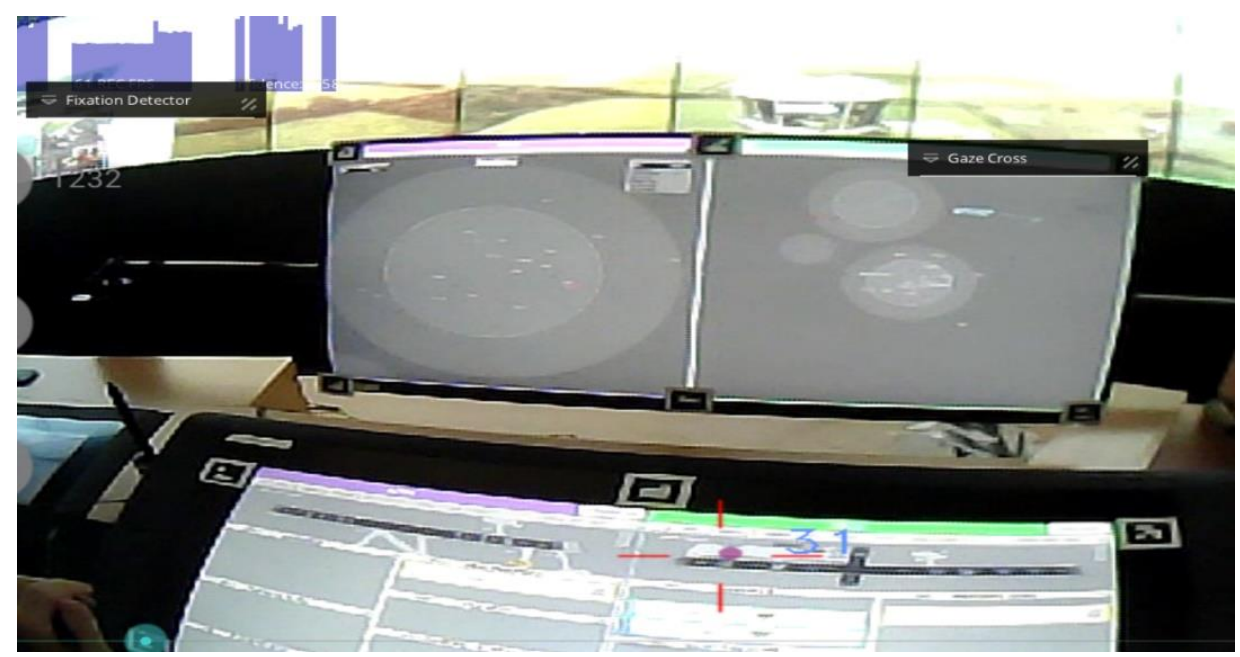

Figure 7. ATCO shifting attention from Shannon to Cork airport to pay attention on the runway activities on the EFS (fixation shown as red-cross recorded by eye tracker)

\subsection{Visual Behaviors Reflecting Complexity of Multiple Tasks}

Fixation numbers and fixation duration are closely linked to each other and are related to cognitive process and human performance (Yu et al., 2016). Short fixation durations primarily indicate operators encoding an element into working memory, and a longer fixation is more likely to signal deeper processing (Ballard, Hayhoe, Pook, \& Rao, 1997). The results demonstrated that visual attention relating to human performance when performing multiple remote tower operations did not exceed the 1,000 millisecond of end-to-end delay, and fitted the requirements of safety assessment (European Aviation Safety Agency, 2015a). To ensure the safety of operations while a single ATCO performing multiple remote tower operations at two different airports and whilst fulfilling the roles of SMC and AMC at both airports, ATCOs found that it would be appropriate to add additional time and lateral spacing between aircraft cleared for take-off so that ATCOs can monitor the roll and initial rotation of the first aircraft before clearing and monitoring a second aircraft for take-off or landing. This finding is important for subsequent operational procedure design.

Eye movements are influenced by the interface design, as information presented by RDP becomes conceptually more difficult, fixation duration increases, and the frequency of regressions increases to process the distance of aircraft for safe separation. Regressions allow ATCOs to revisit previously fixated stimuli such as the text of aircraft call-sign, the figures of flight levels, or images of symbols on the interface displays, and physically returning the eyes to the location of the stimulus could cue the ATCO's memory for that stimulus, effectively aiding the comprehension 
process (Booth \& Weger, 2013). There is a close connection between fixation duration and amount of information processing (K. Rayner, 1998; Singh \& Singh, 2012). When an AMC is managing two simultaneous arrivals into two different airports, ideally the first landing aircraft should be stable on the runway before the second arrival aircraft is 1NM from touchdown at the other aerodrome. However, OTW is the longest fixation duration for SMC due to the nature of complexity of aircraft and vehicle movements in two airports simultaneously. A Single ATCO performing simultaneous AMC and SMC functions is the biggest challenge within MRTO, as RDP and OTW show long fixation durations on SMC plus AMC tasks (figure 4). It requires further investigation to develop effective human-centered design to mitigate the potential risks on multiple monitoring and controlling tasks.

\subsection{Human-Centered Design of CWP sufficiently Support Multi-tasks Performance}

Visual activity is the objective method for assessing an ATCO's cognitive process related to real-time decision-making (Ayaz et al., 2010). The concurrence of excessive fixations, long fixation duration and less saccade duration is the precursor of tunneled attention (Johnson \& Proctor, 2004). ATCO's visual behaviours provide an opportunity to investigate the relationship between eye movement patterns and information processing. Eye scan pattern is one of the most powerful methods for assessing human beings' cognitive processes in Human-Computer Interaction. For example, saccade is defined as fast eye movement between fixations and generally it declines as a function of increased mental workload (Ahlstrom \& Friedman-Berg, 2006). Saccade plays an important role in indicating workload imposed by different tasks among EFS, OTW, RDP and VCS. The results reveal that saccade amplitude has significant interaction between AOIs and tasks (figure 5). ATCOs performing MRTO not only have to distribute their attention to detect potential conflicts among aircraft in the air and on the ground at both Shannon and Cork airports, but also have to resolve unexpected events under time pressure through radio telephony communications with pilots and others.

The VCS is the smallest saccade amplitude among EFS, OTW and RDP consistent with three different tasks. The VCS display consists of a screen with buttons and small digital numbers of radio frequencies used by all moving aircraft, vehicles and other parties on both Shannon airport (on the top of VCS) and Cork airports (on the bottom of VCS). ATCO's must pay attention to select the correct frequency, should an ATCO select an incorrect frequency they may miss transmissions from aircraft/vehicles and may not be able to transmit crucial information to aircraft/vehicles. It demonstrated that ATCOs have more mental workload while interacting with VCS for radio telephony 
communications compared with EFS, OTW and RDP. On the other hand, the OTW is the highest saccade amplitude across tasks. It can be explained that OTW is a good human-centered design to facilitate ATCOs searching required information to perform MRTO by selected ratio of screens to enlarge the images by PTZ. There are lots of Human-Computer Interactions related to the usages of PTZ, as the OTW screens are sufficiently flexible to permit an ATCO to have a larger view of a particular dynamic target to enhance situation awareness (figure 8).

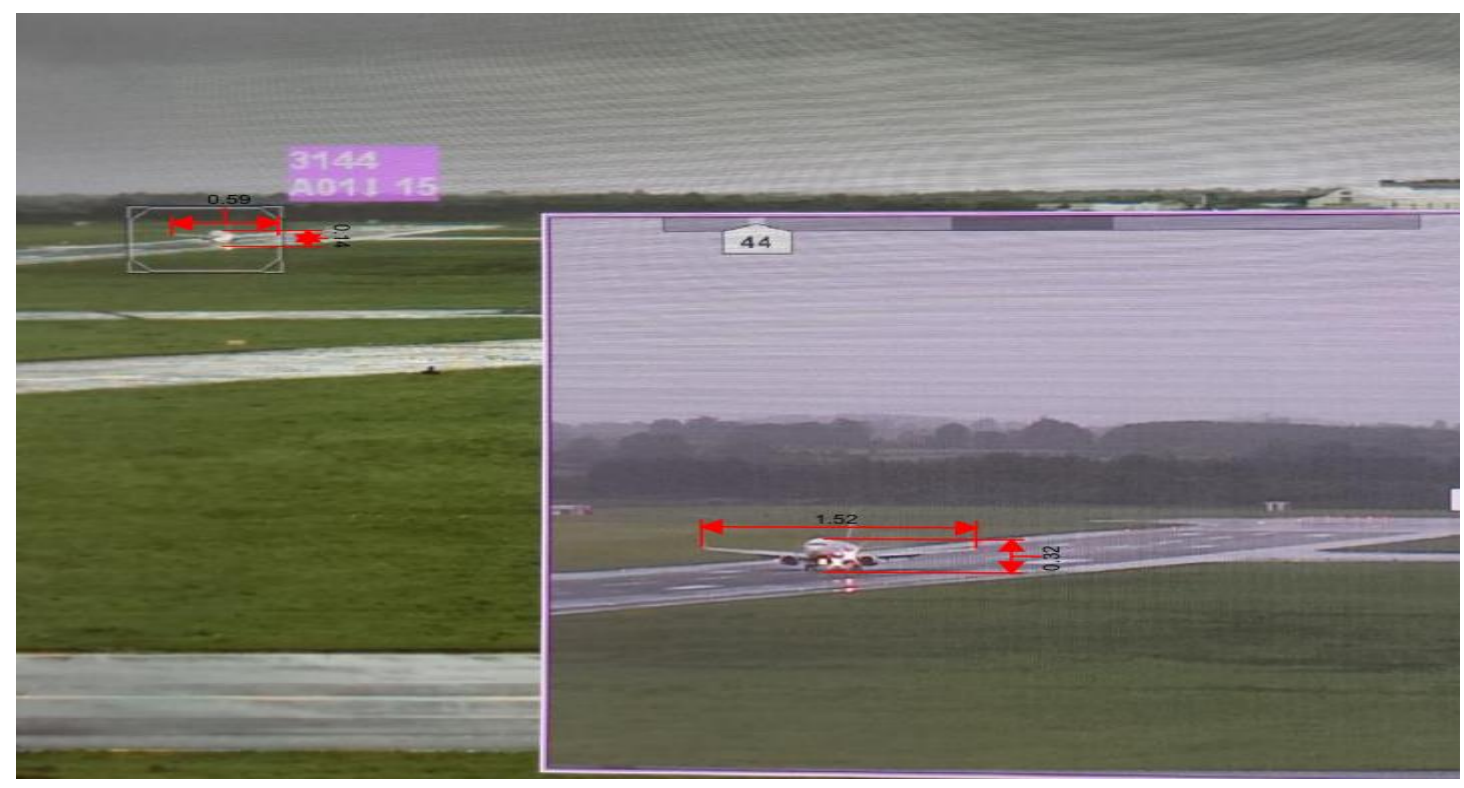

Figure 8. ATCO using PTZ camera to enlarge the visual perception to enhance situation awareness during B-737 Landing

\subsection{Integrated Visual Characteristics with Interface Design Reduced Cognitive}

\section{Loads}

Eye tracking technology offers profound insights into human-computer interaction and the cognitive processes of ATCO's monitoring tasks. The measurement of pupil dilation has been used to investigate the status of cognitive processes and mental workload, as pupil diameter increases as an indication of cognitive demand (Ahlstrom \& Friedman-Berg, 2006). VCS demonstrates the highest pupil dilation compared with EFS, OTW and RDP consistent with AMC, SMC plus AMC and SMC. It is evidence that VCS has induced significant cognitive loads to ATCO on monitoring tasks in selecting frequencies for communications, as ATCO's pupil dilation is the highest and the saccade amplitude is the smallest. This finding supports Ahlstrom and Friedman-Berg's (2006) proposal that 'saccade decreasing mental workload increasing, and pupil dilation increasing cognitive load also increasing'. ATCOs must 
pay attention to select the correct air traffic control frequencies in order to provide effective ATS. Based on the eye tracking data analysis, there are two scenarios $(6.25 \%)$ where an ATCO selected an incorrect frequency on the VCS then realized the errors and corrected them. This indicates a need to investigate how to deal with VCS design to enhance the safety of MRTO. On the other hand, the EFS is the lowest pupil dilation across AMC, SMC plus AMC and SMC (figure 6). This finding implies that ATCOs had the lowest cognitive load while interacting with EFS compared with OTW, RDP and VCS. The different colour borders and runway layout on EFS are very good human-centered design, as it delineates different airports reducing ATCOs' cognitive load and facilitating task performance by clearly defined areas of aircraft information including Arrivals, Pending, Control Zone, Runway, Taxiway, Pushed and Cleared. The dotted-red border on the runway indicates the runway is occupied by a vehicle or aircraft, is used to enhance the ATCOs' situational awareness of activity on the runway and aid in preventing runway incursions. Furthermore, ATCOs are allowed to interact with EFS making notes to support working memory and to serve as reminders for secondary priorities of communication with pilots/vehicle drivers on both Shannon and Cork airports for the deferred responses due to performing multiple tasks (figure 9).

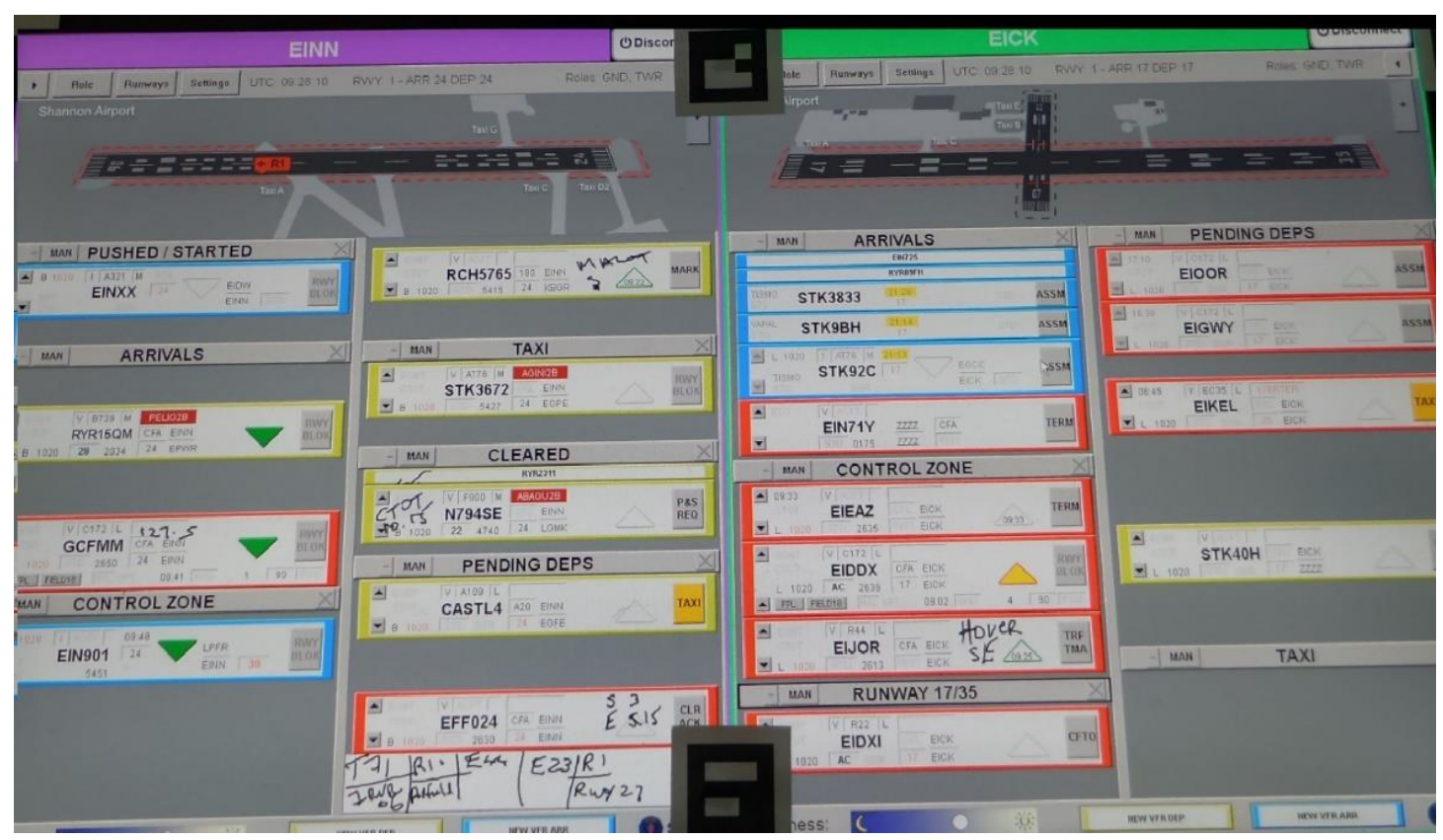

Figure 9. The differential design of colour on the border of EFS increasing ATCO's situation awareness, the Purple colour on the top left indicates Shannon airport, the Green colour on the top right indicates Cork airport

\section{Conclusion and Recommendation}


ATCOs worked with innovative technology and display systems with outside views provided by PTZ camera whilst performing multiple remote tower operations. This research indicates that increased pupil dilation and decreased saccade amplitude in a visual search task are related to strategic adaption to the demands of the tasks for a single ATCO to perform MRTO. The distribution of visual attention among display systems is the key human-computer interaction issue in single ATCOs performing multiple monitoring tasks. Information presentation on the remote tower module and information interpretation by the ATCO are crucial elements in assuring aviation safety and optimal human performance. Current OTW and EFS on the RTM demonstrate that effective human-centred design in relation to information presentation can simplify ATCOs' cognitive processes by reducing the volume of visual searching thereby alleviating cognitive load. Furthermore, innovative remote tower technology will facilitate staffing and equipment cost-efficiencies including Communications, Navigation, Surveillance and Flight Data Processing Systems. There is potential to save approximately €2.21 million Euro per annum per installation. ATCO's visual attention and monitoring performance can be affected by how information is presented, the complexity of the information presented, and the operating environment in the remote tower centre. To achieve resource-efficient and sustainable air navigation services, there is a growing demand to improve the design of human-computer interactions in multiple remote tower technology deployment. These must align with high technology-readiness level, operators' practices, industrial developments, and the certification processes of regulators.

\section{References}

Ahlstrom, U., \& Friedman-Berg, F. J. (2006). Using eye movement activity as a correlate of cognitive workload. International Journal of Industrial Ergonomics, 36(7), 623-636. doi:10.1016/j.ergon.2006.04.002

Allsop, J., \& Gray, R. (2014). Flying under pressure: Effects of anxiety on attention and gaze behavior in aviation. Journal of Applied Research in Memory and Cognition, 3(2), 63-71. doi:10.1016/j.jarmac.2014.04.010

Ayaz, H., Willems, B., Bunce, S., Shewokis, P. A., Izzetoglu, K., Hah, S., . . Onaral, B. (2010). Cognitive workload assessment of air traffic controllers using optical brain imaging sensors. Advances in understanding human performance: Neuroergonomics, human factors design, and special populations, 21-31. doi:10.1201/EBK1439835012-c3

Ballard, D. H., Hayhoe, M. M., Pook, P. K., \& Rao, R. P. N. (1997). Deictic codes for the embodiment of cognition. Behavioral and Brain Sciences, 20(4), 723-742. 
doi:10.1017/S0140525X97001611

Beier, K., \& Gemperlein, H. (2004). Simulation of infrared detection range at fog conditions for Enhanced Vision Systems in civil aviation. Aerospace Science and Technology, 8(1), 63-71. doi:10.1016/j.ast.2003.09.002

Booth, R. W., \& Weger, U. W. (2013). The function of regressions in reading: Backward eye movements allow rereading. Memory \& cognition, 41(1), 82-97. doi:10.3758/s13421-012-0244-y

Carmen Bruder, H. E., Peter Maschke, and Catrin Hasse. (2014). A model for Future Aviation Operators Monitoring Appropriately. Aviation Psychology and Applied Human Factors, 4(1), 13-22. doi:http://dx.doi.org/10.1027/2192-0923/a000051

Chang, Y. H., Yang, H. H., \& Hsiao, Y. J. (2016). Human risk factors associated with pilots in runway excursions. Accident Analysis and Prevention, 94, 227-237. doi:10.1016/j.aap.2016.06.007

Dorighi, N., \& Rabin, B. (2002). NASA uses virtual reality to target runway incursions at LAX. Paper presented at the Federal Aviation Administration Technology Transfer Conference, Atlantic City, NJ.

EUROCAE. (2016). Working group 100 - Developing european standards for remote and virtual towers ED-240.

European Aviation Safety Agency. (2014). Term of reference for a rulemaking task: Technical requirements for remote tower operations.

European Aviation Safety Agency. (2015a). Guidance material on the implementation of the remote tower concept for single mode of operation.

European Aviation Safety Agency. (2015b). Notice of proposed amendment 2015-04: Technical and operational requirements for remote tower operations.

Federal Aviation Administration. (2012). Airport traffic control tower alternatives. Fort Collins.

Friedrich, M., \& Mohlenbrink, C. (2013). Which data provide the best insight? A field trial for validating a remote tower operation concept. Paper presented the 10th USA-Europe Air Traffic Management Research and Development Seminar, Chicago, USA.

Goldberg, J. H., \& Kotval, X. P. (1999). Computer interface evaluation using eye movements: methods and constructs. International Journal of Industrial Ergonomics, 24(6), 631-645.

Hasse, C., \& Bruder, C. (2015). Eye-tracking measurements and their link to a normative model of monitoring behaviour. Ergonomics, 58(3), 355-367. doi:10.1080/00140139.2014.967310

Hasse, C., Grasshoff, D., \& Bruder, C. (2012). How to measure monitoring 
performance of pilots and air traffic controllers. Paper presented at the Proceedings of the Symposium on Eye Tracking Research and Applications, Santa Barbara, California.

Henderson, J. M. (2003). Human gaze control during real-world scene perception. Trends in Cognitive Sciences, 7(11), 498-504. doi:10.1016/j.tics.2003.09.006 Hoffman, J. E., \& Subramaniam, B. (1995). The role of visual-attention in saccade eye-movements. Perception \& Psychophysics, 57(6), 787-795. doi:10.3758/bf03206794

Hollan, J., Hutchins, E., \& Kirsh, D. (2000). Distributed cognition: Toward a new foundation for human-computer interaction research. ACM Transactions on Computer-Human Interaction (TOCHI), 7(2), 174-196.

Honn, K. A., Satterfield, B. C., McCauley, P., Caldwell, J. L., \& Dongen, H. P. A. (2016). Fatiguing effect of multiple take-offs and landings in regional airline operations. Accident Analysis and Prevention, 86, 199-208. doi:10.1016/j.aap.2015.10.005

Irish Aviation Authority. (2016). Remote Towers Demonstration Report: LSD 02.04. Dublin, Ireland.

Irish Aviation Authority. (2017). Annual report of total movements for Cork and Shannon 2016. Dublin, Ireland.

Johnson, A., \& Proctor, R. W. (2004). Attention: Theory and Practice. London: Sage Publications, Inc.

Kassner, M., Patera, W., \& Bulling, A. (2014). Pupil: An open source platform for pervasive eye tracking and mobile gaze-based interaction. Paper presented at the 2014 ACM international joint conference on pervasive and ubiquitous computing: Adjunct publication, Seattle, WA.

Katoh, Z. (1997). Saccade amplitude as a discriminator of flight types. Aviation Space and Environmental Medicine, 68(3), 205-208.

Kearney, P., Li, W.-C., Braithwaite, G., \& Greaves, M. (2017). The Investigation Human-Computer Interaction on Multiple Remote Tower Operations. Paper presented at the 2017 International Conference on Human Computer Interaction, Vancouver, Canada.

Kearney, P., Li, W. C., \& Lin, J. J. H. (2016). The impact of alerting design on air traffic controllers' response to conflict detection and resolution. International Journal of Industrial Ergonomics, 56, 51-58. doi:10.1016/j.ergon.2016.09.002 Koenig, S., \& Lachnit, H. (2011). Curved Saccade Trajectories Reveal Conflicting Predictions in Associative Learning. Journal of Experimental Psychology: Learning, Memory, and Cognition, 37(5), 1164-1177. doi:10.1037/a0023718 Komogortsev, O. V., \& Karpov, A. (2013). Automated classification and scoring of 
smooth pursuit eye movements in the presence of fixations and saccades. Behavior Research Methods, 45(1), 203-215. doi:10.3758/s13428-012-0234-9

Kotval, X. P., \& Goldberg, J. H. (1998). Eye movements and interface component grouping: An evaluation method. Paper presented the Proceedings of the Human Factors and Ergonomics Society 42nd Annual Meeting, Vols 1 and 2, Santa Monica: CA., U.S.A.

Kowler, E. (2011). Eye movements: The past 25 years. Vision Research, 51(13), 1457-1483. doi:10.1016/j.visres.2010.12.014

Kraiss, K., \& Kuhlen, T. (1996). Virtual reality: Principles and applications. In N. Furstenau (Ed.), From Sensors to Situation Awareness. DLR-Mitteilung.

Langan-Fox, J., Sankey, M. J., \& Canty, J. M. (2009). Human Factors Measurement for Future Air Traffic Control Systems. Human Factors, 51(5), 595-637. doi:10.1177/0018720809355278

Lavine, R. A., Sibert, J. L., Gokturk, M., \& Dickens, B. (2002). Eye-tracking measures and human performance in a vigilance task. Aviation Space and Environmental Medicine, 73(4), 367-372.

Leitner, R., \& Oehme, A. (2016). Planning remote multi-airport control- Design and evaluation of a controller-friendly assistance system. In N. Furstenau (Ed.), Virtual and Remote Control Tower: Research, Design, Development and Validation (pp. 139-160). Springer Nature, Switzerland.

Liversedge, S. P., \& Findlay, J. M. (2000). Saccadic eye movements and cognition. TRENDS in Cognitive Sciences, 4(1), 6-14.

Marchitto, M., Benedetto, S., Baccino, T., \& Cañas, J. J. (2016). Air traffic control: Ocular metrics reflect cognitive complexity. International Journal of Industrial Ergonomics, 54, 120-130. doi:https://doi.org/10.1016/i.ergon.2016.05.010

McColemana, C. M., \& Blair, M. R. (2013). The relationship between saccade velocity, fixation duration, and salience in category learning. Visual Cognition, 21(6), 701-703. doi:http://dx.doi.org/10.1080/13506285.2013.844965

Moehlenbrink, C., \& Papenfuss, A. (2011). ATC-monitoring when one controller operates two airports research for remote tower centres. Paper presented at the Proceedings of the Human Factors and Ergonomics Society Annual Meeting.

Muller, T., Giesa, H. G., \& Anders, G. (2001). Evaluation of airborne data link communication. Aerospace Science and Technology, 5(8), 521-527. doi:10.1016/s1270-9638(01)01116-6

Nene, V. A. (2008). A proposed operational concept for nextgen tower. Mitre Corp, Bedford: Mass.

Nickelson, M., Jones, S., \& Zimmerman, T. (2011). Staffed nextgen tower 
human-in-the-loop simulation.

Onken, R., \& Walsdorf, A. (2001). Assistant systems for aircraft guidance: cognitive man-machine cooperation. Aerospace Science and Technology, 5(8), 511-520. doi:10.1016/s1270-9638(01)01137-3

Rayner, K. (1998). Eye movements in reading and information processing: 20 years of research. Psychological Bulletin, 124(3), 372-422. doi:10.1037/0033-2909.124.3.372

Remington, R. W., Wu, S.-C., \& Pashler, H. (2011). What determines saccade timing in sequences of coordinated eye and hand movements? Psychonomic bulletin \& review, 18(3), 538-543. doi:10.3758/s13423-011-0066-0

Robinski, M., \& Stein, M. (2013). Tracking visual scanning techniques in training simulation for helicopter landing. Journal of Eye Movement Research, 6(2).

Salvucci, D. D., \& Goldberg, J. H. (2000). Identifying Fixations and Sccades in Eye-Tracking Protocols. Palm Beach Gardens, U.S.A.

SESAR Joint Undertaking. (2013). SESAR human performance assessment report project ID-06-09-03.

SESAR Joint Undertaking. (2015). Multiple remote tower - Safety assessment report for multiple remote tower.

Singh, H., \& Singh, J. (2012). Human eye tracking and related issues: A review. International Journal of Scientific and Research Publications, 2(9), 1-9.

Van Lancker, J., Mondelaers, K., Wauters, E., \& Van Huylenbroeck, G. (2016). The Organizational Innovation System: A systemic framework for radical innovation at the organizational level. Technovation, 52(Supplement C), 40-50. doi:https://doi.org/10.1016/j.technovation.2015.11.008

Van Orden, K. F., Limbert, W., Makeig, S., \& Jung, T.-P. (2001). Eye activity correlates of workload during a visuospatial memory task. Human Factors, 43(1), 111-121. doi:10.1518/001872001775992570

Van Schaik, F. J., Roessingh, J. J., Lindqvist, G., \& Falt, K. (2016). Detection and recognition for remote tower operations. In N. Furstenau (Ed.), Virtual and Remote Control Tower: Research, Design, Development and Validation. Springer Nature, Switzerland: 53-65.

Wickens, C. D., Helleberg, J., Goh, J., Xu, X., \& Horrey, B. (2001). Pilot task management: testing an attentional expected value model of visual scanning (ARL-01-14/NASA-01-7). Savoy, IL: University of Illinois, Aviation Research Lab.

Yu, C.-S., Li, W. C., Wang, E. M., Braithwaite, G., \& Greaves, M. (2016). Pilots' visual scan patterns and attention distribution during the pursuit of a dynamic target. Aerospace Medicine and Human Performance, 87(1), 40-47. doi:10.3357/AMHP.4209.2016 
Zhao, M., Gersch, T. M., Schnitzer, B. S., Dosher, B. A., \& Kowler, E. (2012). Eye movements and attention: The role of pre-saccadic shifts of attention in perception, memory and the control of saccades. Vision Research, 74, 40-60. doi:10.1016/j.visres.2012.06.017 
2018-05-26

\section{How much is too much on monitoring tasks? Visual scan patterns of single air traffic controller performing multiple remote tower operations}

\section{Li, Wen-Chin}

Elsevier

Li W-C, Kearney P, Braithwaite G, Lin JJH. How much is too much on monitoring tasks? Visual scan patterns of single air traffic controller performing multiple remote tower operations. International Journal of Industrial Ergonomics, Volume 67, September 2018, pp. 135-144 http://dx.doi.org/10.1016/j.ergon.2018.05.005 Downloaded from Cranfield Library Services E-Repository 